\title{
Characterization of effective tire contact area for various tread patterns
}

\begin{abstract}
The effective tire-pavement contact area affects the relative damage of asphalt pavement and should be incorporated in both mechanistic and empirical response analyses of pavements. A new machine called ROTOCOM Wheel Tracker (RCWT) was designed and fabricated to capture the effective tire contact area apart from slab compacting, and conducting simulative laboratory wheel tracking tests. The main focus of this paper is laboratory measurement of effective tire contact areas for various tread patterns. Seven tire treads were selected for the footprint image analyses at five tire loads and four tire inflation pressures. An image processing MATLAB-based program was coded to calculate the contact areas of the 280 imprints obtained from both sides of the RCWT. Factorial analysis indicated significant effects of tire tread, tire load, and inflation pressure on the resulting contact area. Comparison between effective and traditional contact areas indicated that the current pavement design procedure with traditional circular contact area extremely overestimates the actual tirepavement contact area up to $92 \%$.
\end{abstract}

Keyword: Effective contact area; Image processing; ROTOCOM wheel tracker; Tire imprint; Tire tread; Traditional contact area 